\title{
A Comprehensive Survey on WSN Localization, Routing, and Load-Balancing Mechanisms
}

\author{
Mr. Sudhakar K.N ${ }^{1,}$ Dr. Dinesh K Anvekar ${ }^{2}$ \\ ${ }^{I}$ Department of CSE, CMRIT, Bengaluru, E-mail: sudhakar.kn@cmrit.ac.in \\ ${ }^{2}$ Director R \& D / Project Innovation Cell, VVIT, Bengaluru, E-mail: dinesh.anvekar@gmail.com
}

\begin{abstract}
Wireless sensor networks contain various components such as sensing nodes with computational and communicating capabilities. WSN based communication has gained huge attraction from industrial field and researchers due to its significant nature of communication in diverse scenarios in real-time applications such as military application, health monitoring, and civil applications etc. During past decade, ahuge amount of research has been carried out for improving the performance of WSN communication system. Generally, theperformance of wireless sensor network depends on the data collection, routing, and energy consumption minimization. However, these issues have been discussed extensively in previous research but theperformance of WSN is densely deployed environment still remains a challenging task. In this article, we discuss recent studies in the field of WSN. This article presents a review study on WSN routing, WSN localization and load balancing and lifetime enhancement schemes. A comparative analysis is presented based on routing and localization schemes. This study shows that the performance of WSN further can be improved by developing fault-tolerant RSSI based localization, bio-inspired routing algorithms and energy-efficient load balancing schemes.
\end{abstract}

Keywords: WSN, localization, energy efficient routing, load balancing

\section{INTRODUCTION}

In recent years, the low-cost WSNs have gained massive advancements due to development in battery technology, low-power electronics circuits, power-saving schemes, efficient wireless communications, etc. Individually, the WSN is deployed in harsh environmental conditions, in which, the constituting devices must perform selfconfiguration, and involve in cooperative network behavior, along with scalability. On the other hand, the WSN suffers from restricted power and hardware resources, small packet size, data centricity, unreliable communication, functioning without human intervention, random deployment, among many. Due to such characteristics, the WSNs has wide range of applications, including health monitoring, environmental monitoring, terrestrial and underwater monitoring, terror threat surveillance, military surveillance, tracking inventory in warehouses, industrial automation, detecting pipelines corrosion, seismic oil and gas explorations, acoustic detections, process monitoring, homeland security protection, object localization and tracking, disaster detection and prevention, disaster recovery, etc.

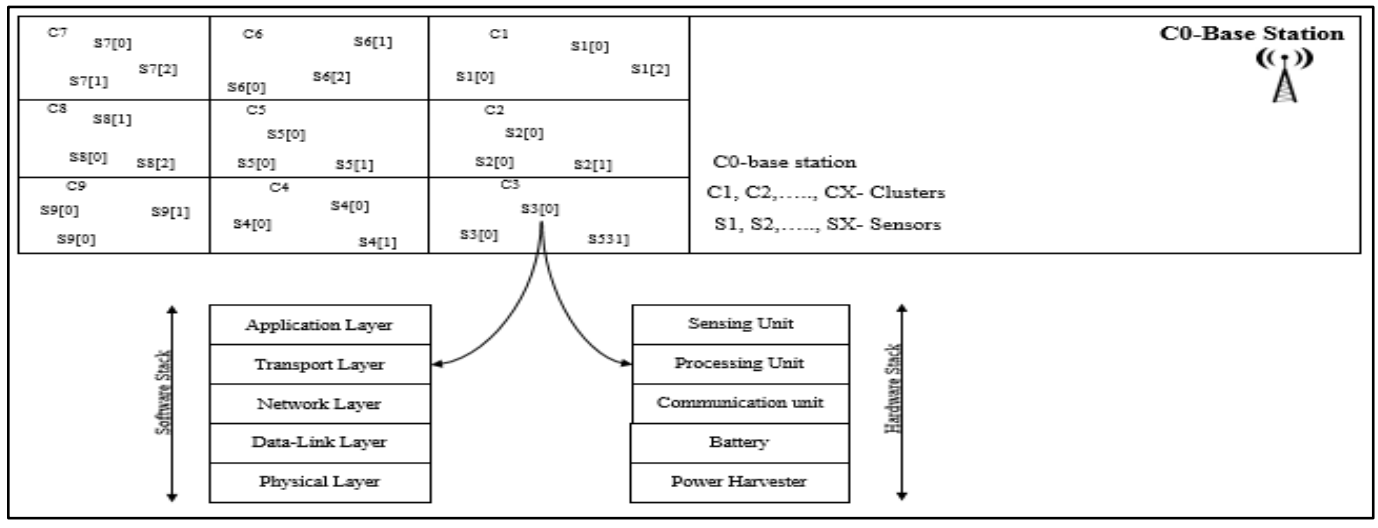

Figure 1: A typical example of WSN architecture 


\section{Available online at www.ijrat.org}

A typical example of WSN architecture is shown in Figure 01. In the figure, each node is composed of a sensing unit which acts as theinput module, a processing unit, a communication unit, along with other supporting resources like a battery, power generator, etc. Akyildiz et al. [1] presented a comprehensive summary of numerous limitations which encourages the design and development of new and fresh WSN. The authors also introduced the WSN software stack, which includes aphysical layer, adatalink layer, transport layer, network layer, and application layer. Over the past few years, there are numerous developments in the various WSN layers. Nevertheless, the research community is still inclined towards the network layer, in particular. As a matter of fact, themajority of the architecture acronyms in the previous and current literature are directly linked to the research and development of the WSN network layer. However, the WSN is not utilized to its full potential due to numerous limitation in the wireless network, and various challenges and hardships involved in design and implementation of WSN.

\section{MOTIVATION AND RESEARCH OBJECTIVE}

Hence, the main objective of this paper is to enhance the overall performance of communication in WSN. Therefore, more importance is given to the three stages of WSN as given below,

1. WSN localization

2. Energy-aware routing

3. Load-balancing.

In the upcoming sections, a brief discussion is made on the latest algorithms, techniques, their merits, and demerits.

\subsection{WSN Localization}

In recent technological development, the IoT or "Internet of Things" has gained immense popularity, because of numerous advantages and scalable features of connecting numerous devices in a given network. Likewise, it has gained the attention of numerous companies and researchers. Constant research and development are made to resolve various problems and issues in this domain. In general, these issues and problems can be segregated into three types, connectivity, sensor, and intelligence. Among them, localization of wireless sensors is one of the areas, which needs significant attention in designing the sensors, computation of limited information and characteristics of wireless channels. Moreover, the location information of the sensor is highly crucial to facilitate other applications in a given wireless sensor networks.

Wireless sensor network has awide range of applications, and they are used in diverse military and consumer application. They are predominant in battlefield surveillance, land-mine detection, environmental monitoring, object detection and tracking, traffic regulation, forest monitoring, etc. In all these applications, the sensor location is quite important. The main objective of the WSN is to identify changes in the given environment and report these changes with aggregated data. The vitality of data depends on the location of the nodes. The location also helps in understanding the application context in the WSN.

There are three main advantages of location information of sensor nodes. First, location information helps in identifying the location of an object of interest. For example, the exact location of fire or disaster, the location of an attacker, location of enemy troops or military tanks in a battlefield, etc. The location information in such domains provides significant data which helps in ado-or-die situation. Second, location information supports various application services. For instance, enemy tanks and troops in a battlefield, target-tracking applications for locating survivors in disaster-hit regions, location directory services which helps the doctors to gain information on nearby medical equipment or expertise in a smart hospital, etc. Third, the location information facilitates numerous system functionalities like geographical routing [2, 3,4], network coverage inspection [5], and location-based data query [6], and many others. Apart from these three advantages, there are numerous benefits of location-aware sensor nodes, because of which, they have become a standard equipment in most WSNs.

A simple technique to embed GPS transceiver on each sensor can help the sensors to accurately estimate their current location. Nevertheless, this technique is slightly expensive, and it is not entirely suitable for economic or low-cost networking applications. As the network contains thousands of sensor nodes, it is very costly to equip each sensor node with individual GPS module. Furthermore, manually configuring the location for each and every node is time-consuming and ineffective. Thus, localization has grown into a critical problem in WSNs. In recent years, several protocols were developed to assist the nodes to extract their location information in WSNs. The process can be carried out autonomously without any additional hardware or human intervention $[7,8,9,10,11]$. In these techniques, the location information is extracted with the help of special nodes, known as beacon nodes. The beacon nodes are also called as alocator, anchor node, seed node, etc. When localization is considered, there are three main parameters, accuracy, energy efficiency, and security. Numerous research has been conducted in this regard, but the outcomes suggest that more intense research is required to improve the performance of the localization in WSN. Therefore, a brief review is presented on various localization techniques. 


\section{E-ISSN: 2321-9637 \\ Available online at www.ijrat.org}

\section{i. Challenges in WSN}

In comparison to other networks, the WSN is implemented in regions which are harsh unknown. The WSNs experiences numerous challenges which are normally not encountered by traditional networks, such changing temperature, hot, snow, rain, storms, dust, wind, humidity, etc. The situation is much worse in amilitaryapplication, involving target tracking, battlefield surveillance, etc., where the network has to witness landmines, attacks, etc. While operating under such influential environmental conditions, the WSNs must perform autonomously and withstand all incoming challenges and issues. On top of that, an external attacker can steal access to the network and may physically damage the constituent devices, mainly the nodes. Once a node falls under the influence of the adversary, the oncoming events will not be favorable. Using the captured node, the adversary can broadcast malicious or corrupted data in the network. This results in unnecessary resource wastage and causes malfunction.

Moreover, the confidentiality and secrecy of the genuine data are also prone to therisk of theft. After gaining control over the network, the adversary can launch several attacks on the network as an insider. In this stage, themajority if the networks will fail. Let's consider a beacon-based localization model for example. In this model, the nodes are unaware of their current location. They depend on beacons sent by other nodes to determine their location. But, since the network is under the influence of an adversary, the node cannot decide if the beacon is reliable and accurate. To be more precise, the adversary may intentionally send out a false beacon, which results in miscalculation of location. This results in variation of information, which is termed ad information asymmetry. The information asymmetry in beaconbased localization models is presented in ref. [12]. Ref. [12] also introduces some techniques to address insider attacks. Depending on the intensity to disturb the localization, an attacker can instigate replay attacks, wormhole attacks, Sybil attacks, etc.

\section{ii. Overview of WSN localization}

The localization process can be segregated into two stages. In the initial stage, the node utilizes the received signal to compute its distance from other nodes in its neighborhood. In the next stage, a node estimates its actual location using all the distanceb) metrics. In this second stage, the technique used to estimate the actual location relies on the signal feature employed in theprevious stage. This can be segregated into three classes as given below.

(a) Triangulation: Majority of the localization algorithms comes under this category. In general, the triangulation techniques consists of capturing Angle of Arrival $(A o A)$ measurements of a signal at the sensor node arriving from at least three different
Localization is a process of estimating the current location of the sensor nodes. It generally involves estimating the spatial relationships between numerous objects in a given plane. There are numerous localization techniques inseveral kinds of literature, each of which has their own assumptions regarding the network and node abilities. Some of the commonly made assumptions are signal propagation models, cost of communication, device hardware, operational environment (indoor, outdoor, underground, underwater, etc.), error requisites, energy requisites, timing constraints, time synchronization, network contention, such as homogeneous vs. heterogeneous, beacon density, and node mobility [11]. There are different scenarios in node mobility,

(a) Both beacon and sensor nodes are static.

(b) Beacon is mobile, and, sensor nodes are static

(c) Beacon is static, and sensor nodes are mobile.

(d) Both beacon and sensor nodes are mobile.

When the source uses GPS in localization models, then localization process is simple and direct. Alternatively, when the localization model uses beacon nodes to determine the location of other sensor nodes, then the beacon nodes must be configured manually or they must be embedded with GPS transceiver. Once the beacon node knows their location, they share its location information with other nodes to determine their location. Figure 2 presents the basic working of beacon-based localization.

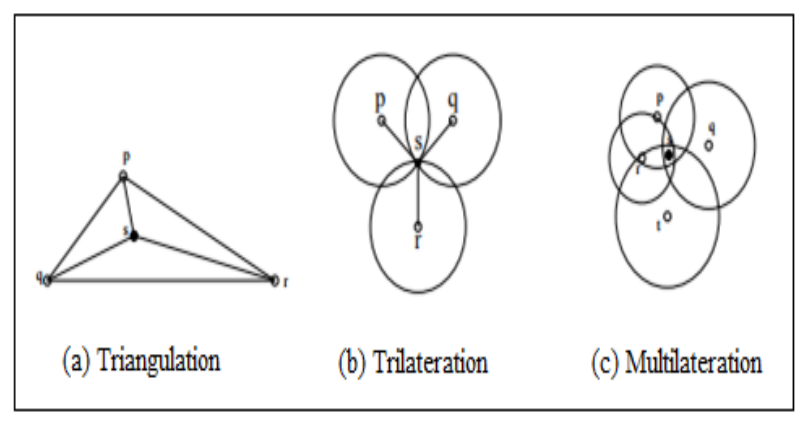

Figure 2: localization technique

sources. With the help of AoA references, generic geometric relationships and properties can be implemented to estimate the current location of a sensor node.

Trilateration: Trilateration is a process of using thegeometry of triangles for estimating the relative positions of objects. It is almost identical to triangulation. While the triangulation process uses AoA measurements to estimate the location of an object, the trilateration aggregates a number of reference tuples in the form of $(x, y, d)$, for the same purpose. $(x, y)$ Represents the some reference location, and $d$ gives the estimated distance from this reference point to a sensor node. In general, at least 3 


\section{E-ISSN: 2321-9637 \\ Available online at www.ijrat.org}

reference points are required for precise and accurate estimation of relative location of a point on a 2Dimensional plane using trilateration.

(c) Multilateration: Multilateration is a more complex process of localization, which involves solving for the mathematical intersection of multiple hyperbolas on the basis of the Time Difference of Arrival (TDoA). Multilateration is applied to the TDoA of a signal forwarded by a sender to three or more receivers. It can be accurately calculated using tightly synchronized clocks. If there are $\mathrm{N}$ receivers, then there will be $N-1$ hyperbolas. The intersection of the parabolas of an object can be easily formulated over a 3-Dimensional plane. When the number of receivers are high, then the localization problem can be considered as an optimization problem. In this case, a least squares method can be used to solve the optimization problems.

\section{iii. Classification of WSN localization}

In this section presents various techniques utilized for localization of WSN. The merits and demerits of the techniques are also highlighted.

(a) Direct approaches: This approach is also called as absolute localization. They are categorized into two types, namely, GPS-based localization and Manual configuration. In the GPS-based localization, the sensor will be embedded with GPS transceiver. The GPS helps in determining theexact location. This technique support node mobility. The manual configuration approach is highly complex, cumbersome and doesn't come cheap. This approach does not support scalable feature for large WSNs. It also does not support the node mobility. However, the GPS based approach also suffers from few drawbacks. On of prominent is the cost of equipment. It is expensive to install and maintain GPS modules for each sensor nodesbecause, in some networks, there can be thousands of nodes. It also utilizes more battery power and increases the size of the nodes hardware. There is also an issue with theconnectivity of GPS in closed or confined areas. This approach is not suitable for underground, underwater applications.

(b) Indirect approaches: The indirect localization techniques are also called as relative localization approaches. This is due to the fact that the position of the nodes deployed with respect to other nodes in their neighborhood. The indirect localization schemes are designed to address and challenges and problems associated with the disadvantages and limitation of GPS-based direct localization scheme. However, certain benefits of direct localization scheme, such as accuracy, precision, etc., are retained. In indirect localization techniques, a small subset of nodes in the network is aware of their location information, either through manual configuration or by embedding a GPS module. Such nodes are called as beacon nodes. The beacon nodes forward signal beams, which provide their location information to all other neighboring sensor nodes, who cannot determine their location on their own. With the help of location information present in the signal beam, sensor nodes can estimate its own location. The indirect localization techniques considerably minimize the complexity encountered in theGPSbased method. Nevertheless, there is certain risk associated with indirect localization techniques. As the beacon nodes are also present in the same harsh environment, they too face same dangers as experienced by other normal sensor nodes. The beacon nodes are also susceptible to numerous attacks, threats, and physical damages. This raises the concern about the security threats of the beacon nodes. If a beacon node is compromised, then the location information forwarded by that nodes will be inaccurate or corrupted. This results in theerroneous computation of location. Ref. [13] has taken measurements to address this issue. In their technique, a trust and reputation based system is used to check for such malicious beacon nodes and misbehavior. Generally speaking, the localization process can be categorized into two types.

(c) Range-based: In range-based localization, a node estimatesits location based on its relative distance from other nodes in its neighborhood. This technique makes an assumption that the unconditional distance between a source and a destination can be computed by one or more parameters of the transmitted signal. The network environment and transmission medium affect the precision and accuracy of such computations. Range based localization schemes generally depend on complex hardware, which cannot be embedded in WSN devices, due to the resource constraints of the constituent sensor nodes. Moreover, the large number of sensor nodes makes it expensive to implement such hardware on sensor nodes. Some of the well-known range-based localization schemes are given in ref. [14, 15, 16, 17]. The range-based localization schemes consider certain parameters of thecommunication signal, which are presented below,

Angle of Arrival (AoA): Range information can be estimated by computing and mapping relative angles between the neighboring nodes. The AoA parameter is used in ref. [18,19] for localization.

Received Signal Strength Indicator (RSSI): The distance between source and receiver can be determined from the received signal strength using theoretical or empirical model. Such a technique was first used in D-Log [20]. This parameter is also used in ref. [21, 22] for range estimation.

Time of Arrival (ToA): The range information can also be obtained by computing the time taken for a signal to reach from source to destination. This parameter is predominantly used in GPS technology. However, to utilize ToA information for range 
calculation, the sender and receiver must be synchronized. This demands expensive hardware to provide accurate clock synchronization with the satellite. Ref. [23, 24] uses ToA for localization.

$\checkmark$ Time Difference of Arrival (TDoA): The range estimation using TDoA can be performed by using an ultrasound transceiver to compute the visible distance from thesource to destination. This additionally requires expensive hardware, which is not always suitable for most WSNs. Some the literature which uses TDoA are given in ref. [25, 26, 27].

(d) Range-free: Range-free localization doesn't depend on the received signal strength or other parameters of thereceived signal, such as angle of arrival, time of arrival, etc. Hence, it doesn't compute absolute point-to-point distance. This eliminates the need for complex hardware. Furthermore, this approach becomes cheaper to implement. It can be utilized an alternative approach for WSN localization. Some of the popular examples of range-free localization techniques areamorphous localization [28], Centroid localization [29] and DV-Hop localization [30].

On the basis of various types of localization techniques, a comparative analysis is presented in table 01. The anchor free, anchor based, range free, range based, distributed and centralized techniques are compared for comprehensive analysis.

Table 01: Comparative analysis based on localization classification

\begin{tabular}{|l|l|l|l|l|}
\hline \multicolumn{1}{|c|}{ Authors } & \multicolumn{1}{|c|}{ Year } & \multicolumn{1}{c|}{$\begin{array}{c}\text { AnchorFree/Anchor } \\
\text { Based }\end{array}$} & Range-Free/ Range Based & \multicolumn{1}{|c|}{$\begin{array}{c}\text { Distributed/ } \\
\text { Centralized }\end{array}$} \\
\hline Zhang et al [32] & 2017 & AnchorBased & RangeFree & Distributed \\
\hline Heng [33] & 2017 & Anchor Free & Range Based & Centralized \\
\hline Zhao et al. [34] & 2017 & Anchor Based & Range Based & Centralized \\
\hline Li [35] & 2017 & AnchorBased & Range Based & Centralized \\
\hline Singh [36] & 2017 & AnchorBased & RangeFree & Distributed \\
\hline Porta [37] & 2016 & AnchorBased & RangeFree & Centralized \\
\hline Pivato [38] & 2011 & Anchor Based & Range Based & Centralized \\
\hline Zhang [39] & 2015 & AnchorBased & RangeFree & Distributed \\
\hline Kwon [40] & 2014 & Anchor Based & Range Based & Centralized \\
\hline Tran [41] & 2008 & AnchorBased & RangeFree & Distributed \\
\hline
\end{tabular}

\section{iv. Comparative study of localization algorithms}

(a) Performance Parameters

1) Beacon/Anchor Nodes: Beacon nodes are special nodes which are aware of their location in a given space. They are usually equipped with GPS module to determine its location, or it can be manually configured. The localization process is strongly influenced by the beacon configuration. Table 1 presents acomparative analysis of few beacon based algorithms. These approaches have few demerits. The foremost one is the cost, which is high due to GPS and other hardware for location detection. Moreover, such nodes are rendered useless in underground, underwater and indoor applications.

2) Node Density: Most of the localization algorithms are sensitive to node density. For instance, the hopcount based algorithms demand high node density in order to make acorrect estimation of a number of hops approximating for a given distance [26].
Therefore, during thedevelopment of an algorithm, it is crucial to determine the requirements of the algorithm with respect to node density.

3) Accuracy: Accuracy is a significant parameter of any Localization algorithm. In certain application, the WSN will be installed to carry out acritical operation such as rescue, search, detection/tracking, etc. In such cases, the algorithm should be provided with theaccurate location of the nodes to avoid any miscalculation, which can be fatal.

4) Cost: Generally, the cost of a localization algorithm is mostly composed of communication cost, hardware cost, and computation cost. Communication cost includes two segments, (1) communication between nodes and Base Station, and (2) inter-node communication. Hardware Cost mainly contains node density, which is the density of reference nodes and measuring device [26]. Centralized Localization process occasionally 


\section{Available online at www.ijrat.org}

includes communication between sensor nodes and BS. Sadly this communication cost with respect to energy utilization is higher in comparison to internode communication. This the main reason why distributed algorithms are more appropriate than centralized algorithms. In distributed schemes, the inter-node communication is more complex than communication between BS and nodes.

Table 02: presents the comparative analysis of range free and range-based localization algorithms.

\begin{tabular}{|c|c|c|c|c|c|c|}
\hline $\begin{array}{c}\text { Localization } \\
\text { Approach }\end{array}$ & Modus operandi & $\begin{array}{c}\text { Localization } \\
\text { Accuracy }\end{array}$ & $\begin{array}{c}\text { Computation } \\
\text { cost }\end{array}$ & $\begin{array}{c}\text { Hardware } \\
\text { cost }\end{array}$ & $\begin{array}{c}\text { Communicatio } \\
\text { n cost }\end{array}$ & $\begin{array}{c}\text { Coordinate } \\
\text { s }\end{array}$ \\
\hline \multirow{5}{*}{ Range Based } & \multicolumn{6}{|c|}{ Operates using distance based techniques } \\
\hline & RSSI & Medium & Low & Low & - & - \\
\hline & TDoA & High & High & Low & - & - \\
\hline & \multicolumn{6}{|c|}{ Angle Based technique } \\
\hline & AoA & High & High & Low & - & - \\
\hline \multirow{5}{*}{ Range-Free } & \multicolumn{6}{|c|}{ Hop Count Based Technique } \\
\hline & Per-hop distance & Medium & - & Low & - & - \\
\hline & \multicolumn{6}{|c|}{ Neighborhood-Based Techniques } \\
\hline & Single neighbor & Low & - & Low & - & - \\
\hline & Multi neighbor & Low & - & Low & - & - \\
\hline Centralized & - & High & High & - & High & - \\
\hline Distributed & - & Medium & Medium & - & Low & - \\
\hline Anchor based & - & High & - & Very high & - & Global \\
\hline Anchor Free & - & Medium & - & Very low & - & Relative \\
\hline
\end{tabular}

\section{Summary of localization performances}

It has been previously established that current localization schemes are generally introduced to fulfill the requirements of diverse application requirements. However, it is not very easy to compare the results, as it is intensified through various test-beds that are created for experimentation and evaluation. Nevertheless, it is not entirely impossible to review the performance of solutions pertaining to communication, accuracy, energy consumption, node density, and computation costs. Table 2 presents the localization accuracy as evaluated based on the compromise among communication, accuracy, energy consumption, node density and computation costs, node deployment, etc. Besides randomly deployed networks, the nodes can also be deployed in a grid of non-anchor nodes inside a specific region. The precision and accuracy of localization are generally computed using theaverage Euclidean distance between the actual locations and the estimated locations which are normalized to transmission range. In case of mobile-based localizations, the influence of node density does not contribute much towards the localization of static nodes. Moreover, computation costs are not much significant for experimental simulations as opposed to practical implementations.

\section{Open Issues}

Over the past few decades, several improvements and research have been carried out in sensor localization. Even then, there are numerous other issues which are still not entirely resolved.Such issues are listed below,

$\checkmark$ Energy consumption:Several researchers have worked on energy consumption in thelocalization of WSNs and introduced numerous algorithms to 
address this issue. But, developing an energy efficient localization technique is not an easy task. Developing energy efficient schemes at the various communication layers of aprotocol stack for localization technique in WSN needs asuitable analytical model for quantifying energy utilization. Noticeably, this is a significant process, as it covers numerous unrelated process like communication with neighboring nodes, localizationrelatedmeasurements, and computing locations.

$\checkmark$ 3-Dimensional WSNs: In most application, the localization schemes in WSN estimates the location of nodes in a2-dimensional plane. But in some applications, nodes should be deployed in a3dimensional plane. This causes asignificant offset in both ranging results and localization approaches. Therefore, localization schemes for 3-D space are more important real-time WSN applications, particularly when there is a huge difference between localizations in 2 dimensional and 3dimensional planes. For example, variations in radio transmission are considered in $2 \mathrm{D}$ space, whereas, it not well researched in 3D space.

$\checkmark$ Security and privacy: One of the critical issues in thedeployment of WSNs is the privacy and security. Alternately, in few other application, accurate locations information of nodes are more important. In such cases, revealing this location information to other external applications may lead to unforeseen circumstances which can compromise the network and hampers the data security. This can also lead to external attacks. There is numerous literature on thesecurity of localization [42, 43], but the types of attacks experienced by those approaches and the related preventive measures taken to avoid such attacks, are still not asserted properly.

\subsection{Energy-aware routing in WSN}

In some applications, the sensor nodes in the network are left unattended. Such habit strongly influences the performance of efficiency of the application. Some of the affected application areas are, intrusion detection, target field imaging, consumer applications, military applications, weather monitoring, industrial surveillance, tactical surveillance, stock management, distributed computing, temperature monitoring, environmental monitoring, disaster management, etc. In most of this kind of applications, the nodes are deployed randomly. They can be dropped off from thesky, or manually installed at some preset locations, etc. In adisaster-hit area which requires WSN installation, the nodes can be dropped randomly from helicopter or airplane. After deployment, the nodes will configure themselves and monitor the area to check for any survivors to assist and fasten the rescue and recovery process. They might also act as an emergency communication system.

In the past couple of years, a comprehensive research was conducted to resolve the issues pertaining to acooperative effort among sensors in information aggregation, processing, and communication. Nevertheless, the sensors have restricted amount of energy and other resources. Subsequently, new strategies and mechanisms are needed, which limit the energy wastage and lead to enhancement of network lifetime. Along with such limitations, a conventional sensor deployment in high quantity may also impose numerous problems to the network design and implementation. This demands the need for more efficient networking protocol stack with energy-aware schemes for all layers. For instance, at the network layer, it is profitable to implement new techniques for energyefficient route establishment and forwarding data from the sensor nodes to the BS, in such a way that the network lifespan is greatly enhanced.

The intrinsic characteristics of WSN make it a highly challenging area to design and implement efficient routing schemes. The WSN characteristics are very different from any other wired or wireless networks, such as mobile ad hoc networks or cellular networks. First, the number of nodes in the network is very high. This creates a problem in creating a global addressing scheme for mass deployment of sensor nodes in high volume. This is not possible and requires individual addressing, which imposes an additional overhead for maintaining the identities of nodes. Hence, conventional IP-based protocols are not compatible with WSNs. Additionally, the sensor nodes which are deployed randomly in an ad hoc fashion must be self-governing and should install itself, independently. The ad hoc deployment of the nodes dictates that the system has to establish connections among the nodes and adapt to resultant node distribution, particularly for functioning independently. In most WSN application, data transmission is more crucial than getting the identity information of the nodes.

Second, compared to other traditional networks, the WSN applications demand that the data has to be collected from multiple sensors, and forward all the sensed data to a base station. This does not eliminate the data flow in other forms, such as peer-to-peer, multi-hop or multicast. Third, the sensor nodes have limited hardware resources, such as low energy, low computation power, limited memory and storage capacities, etc. Therefore, the node resources must be carefully managed. Fourth, in themajority of WSN applications, the nodes are typically considered as static after deployment, however, there might be some cases where few modes will be mobile. In 
few other wireless networks, the nodes are free to move inside the confined area, which causes sudden changes in network topology and unforeseen behavior. Even then, if mobility is a critical requirement of the application, the mobile nodes are allowed to move about.

Fifth, most sensor networks are application specific, which signifies that the design requisites of a sensor network vary from one application to other. For instance, the requirement of industrial monitoring is different from battlefield surveillance. Sixth, some application requires location information of nodes, as they are needed for collecting data according to aparticular location. The GPS (Global Positioning System) is the most accurate form of location detection but is not costeffective to use in most WSN applications. There are other methods, such as triangulation [44], which can help the nodes to roughly estimate their location based on the received signal strength from other location-aware nodes. It can be observed in ref. [44], that the techniques based on triangulation or Multilateration can greatly assist in estimating the location of a node using other location-aware nodes. Few nodes are aware of their location either through GPS or manual configuration [9]. Such techniques eliminate the need for costly equipment in WSN. Finally, the data captured by numerous sensors in a network are based on common criteria and somewhat identical environment. This suggests that there are chances that the nodes generate same data, which creates data redundancy. Data redundancy can degrade the network, and hence routing protocols must eliminate the redundancy and make the data transmission, more efficient.

Almost all the WSNs are data-centric networks. It implies that the network demand for data to be sensed on the basis certain condition, which is known as attribute-based addressing. It consists of a query set with a pair of attribute and value. For instance, a simple query will be given in the form of [temperature $>60 \mathrm{~F}$ ]. The sensor nodes considered the $60 \mathrm{~F}$ as athreshold and senses the temperature which is higher than $60 \mathrm{~F}$. This gives rise to numerous problems, because of which, several routing algorithms are proposed for WSN. These routing schemes consider the intrinsic characteristics of WSNs without deviating from theapplication and architectural requirements.

The process of discovering and establishing paths in WSNs is highly significant as there are numerous resources limitations and drastic variations in node status (online, offline, idle, failure), which results in recurrent and random topological changes. In order to reduce the energy utilization, several routing algorithms are introduced in the literature for WSNs. These algorithms utilize few popular routing schemes of
WSNs, such as clustering, data aggregation, diverse node role assignment, in-network processing, datacentric methods, etc. The prevailing routing algorithms can be categorized based on network structure as, hierarchical, flat or location-based algorithms. In hierarchical routing protocols, different nodes perform different operations and function. It is done by clustering the network, in order to eliminate redundancy and save energy. In case of flat routing protocols, all nodes perform identical operations.

Additionally, the routing protocols can also be categorized based on protocol operation, which is, query-based, multipath-based, QoSbased, negotiation-based, and coherent-based. In location-based protocols, the algorithm uses location information of the nodes to forward the data to the designated sink, instead of randomly flooding the entire network. The following section discusses briefly these routing protocols and latest advancements in these fields. Later, each of this protocol will be discussed. The main aim is to provide a good level of understanding about these protocols and other research in this regard.

\section{Routing challenges and issues in WSN}

Wireless sensor networks have numerous applications in military, industrial and consumer segments. But they suffer from several limitations such as restricted resources, lower power, limited energy, lower bandwidth, etc. In any WSN, irrespective of its size or application, the main objective is to perform reliable data communication without shortening the lifespan of the network. Additional emphasis is laid on reducing connectivity issues by using proficient energy management schemes. While designing routing protocols in WSNs, several factors must be considered. It is essential fulfilling these factors prior to achieving energy efficient communication in WSN. In the following sections, a discussion is made on some of the major challenges faced in the design of energy efficient routing in WSN.

$\checkmark$ Node deployment: Node deployment in WSNs depends on the type of application. It has a profound effect on the performance of the protocol. The deployment can be random or deterministic. In random deployment, the sensor nodes are placed randomly which establishes an ad hoc network. In deterministic deployment, the sensor nodes are placed manually. In this technique, the data is also transmitted using pre-determined routes. When the node deployment is not distributed uniformly, then clustering technique must be implemented to make way for reliable connection and assist in achieving good levels of energy efficiency. Inter-sensor communication takes place for only short transmission ranges because of bandwidth and 
energy restrictions. Hence, it is considered that routing takes place through more than one nodes with numerous hops.

$\checkmark$ Energy consumption without affecting the accuracy: Sensor nodes have limited supply of energy. And most of this energy will be exhausted for sensing data, processing, and data transmission. Therefore, there is a requirement for energy-aware data processing and communication. The uptime of the sensor node is strongly dependent on the battery backup [45]. In a multi-hop WSN, the node acts as both sender and router. When a node is turned off due to battery drainage, then the node is said to be failed. It imposes serious repercussions on the topology of the network, as the path joined by that node will also fail. Therefore, the network must be configured all over again, with creating new alternative paths to all the failed routes.

$\checkmark$ Data Reporting Model: The type of application and the time criticality of the WSN dictates the method of data sensing and reporting. Data reporting can be classified into four types, querydriven, event-driven, time-driven (continuous), and hybrid [46]. In query-driven and event-driven models, the sensor nodes respond to radical and sudden variations in the value of a sensed data, with an intention that such change might be triggered due to some external event or action. Therefore, such models are suitable for timecritical applications. The routing protocol is strongly affected by data reporting model with respect to route stability and energy utilization. On the other hand, time-driven models are appropriate for applications that need occasional data monitoring. In these models, the data will be sensed and transmitted periodically at regular intervals of time.

$\checkmark$ Node/Link Heterogeneity: In themajority of WSN application, the nodes considered to be homogeneous. In other words, the nodes are considered to possess theidentical capacity of energy, processing power, computation, memory, etc. Nevertheless, such capabilities among different sensor nodes will be different in few kind of application. Such variation in sensor node creates a heterogeneous network, which imposes another set of challenges and issues. For instance, few applications need a variable combination of sensors for monitoring pressure, temperature, and humidity of the environment. Some application includes detecting motion through their acoustic signatures. These applications are also used in object detection and tracking. Therefore, some sensor nodes will have additional capability over other sensor nodes. Such enhanced sensor nodes can be deployed individually. Such sensor nodes might also generate and transmit data at adifferent rate compared to normal nodes. It might be faster or slower, depending on the application requirement, service criteria, etc. For instance, in hierarchical protocols, a cluster head is designated with themuch-sophisticated task than other normal sensors. Cluster heads are selected from the deployed sensors which contain higher residual resources, such as, memory, energy, computational power, etc. Additionally, the transmission of data to base station must also be carried out by the cluster heads.

$\checkmark$ Fault Tolerance: Few sensor nodes may occasionally encounter failure or blockage due to physical stress, limited energy, or other environmental conditions. However, such node failure should not degrade the performance of the network. In case if several nodes fail, then the routing algorithm and MAC protocol should make analternative arrangement by establishing new paths to route data from source to destination. This demands dynamic tuning of transmit powers and signal transmission rates on live paths to minimize energy usage. The packets can also be rerouted through other regions of the network where more energy is available. Hence, for a proper faulttolerant network, multiple levels of redundancy might be advisable.

$\checkmark$ Scalability: In a typical WSN, there will be hundreds, sometimes even thousands of sensors nodes. Therefore, it is essential for a routing algorithm to handle such large amount of nodes, and work accurately and efficiently. Moreover, the sensor network routing schemes must be scalable to adapt itself to changes in thenetwork or any other events triggered in the environment. However, the nodes can continue being in sleep mode, until an external event arises. The rest of the nodes can carry out their task with reasonable quality.

$\checkmark$ Network Dynamics: In general, the nodes in the network are assumed to be stationary. But in fewapplications, acertain amount of mobility is needed for few nodes, and even the base station [19]. In such cases sending or receiving messages to/from such mobile nodes are highly difficult. The route connecting to mobile nodes are always at the risk of breakage. It uses more bandwidth and energy. Furthermore, the sensed data can be static or dynamic, which is purely dictated by the type of application. For example, an object detection and tracking application, the data is dynamic. In environment monitoring, the data collection is static, as it is collected from certain points of interest. When the nodes work with static data, they tend to be reactive in nature, which creates unnecessary traffic. In case of dynamic events, the data is collected periodically. In such cases, the aggregated data is sent to base station simultaneously, which increases the traffic for a certain amount of time. 
Transmission Media: Communicating nodes in a multi-hop sensor network will be linked through awireless medium. This causes certain drawbacks with respect to conventional challenges encountered by a wireless channel, such as signal fading, high error rate, etc. The required bandwidth of sensor data will be generally lower, say 1-100 $\mathrm{kb} / \mathrm{s}$. As far as transmission media is concerned, a medium access control (MAC) design is necessary. Bluetooth is cheaper and affordable technology for this purpose. Additionally, more complex techniques like TDMA can also be used for MAC design, which is proven to be more efficient than other CSMA protocols.

$\checkmark$ Connectivity: Due to high node density in most WSN, the nodes are not entirely isolated from one another. They are always connected and always available. This does not guarantee stability in network topology, as it may change anytime. The network size is also varied depending on the connectivity. Generally, connectivity is determined by therandom distribution of nodes.

$\checkmark$ Coverage: In any WSN, the sensor nodes have a definitive view of the surrounding environment. This view of the sensor will be limited in terms of accuracy and range. The extent of coverage area will be limited. Thus, coverage area plays an important role in WSN deployment.

$\checkmark$ Data Aggregation: It is prevalent that sensor nodes create alarge amount of redundant data. It is sensible to aggregate the data from all the sensor nodes and eliminate the redundant data. If such redundancy is not removed, then redundant data transmission will result in unwanted energy usage. Data aggregation is a process of collecting data from different nodes with respect to a specific aggregation condition. For instance, minima, maxima, duplicate suppression, and average. This approach is implemented to attain good levels of energy efficiency and efficient data transfer. Data aggregation can also be accomplished using Signal processing techniques. In such situations, it is considered as a data fusion, in which a node is able to generate anoutput signal with high precision. Good levels of accuracy can be achieved using beamforming techniques to combine the incoming signals and minimizing the noise and distortion in them.

$\checkmark$ Quality of Service: In certain WSN application, there is a stipulation that the sensed data should be forwarded within a certain amount of time. Failure to meet this criterion will render the data useless. Thus, thetime delay for data delivery is an important criterion for most time-constrained WSN applications. In other application, the principle criteria will be energy saving, which eventually translates to extended network lifetime. This is considered more important than any other quality attribute in WSN. When the network experiences lower energy levels, the quality of data sensing and communication is also hampered. This is intentionally done to reduce unwanted energy consumption in nodes and extend the overall network lifetime. Therefore, energy-aware routing protocols are essential to fulfillingthis criterion.

\section{Routing protocols in WSN}

In this section, a survey of popular routing protocols in WSNs are presented. Based on the network structure, the routing protocols in WSNs can be categorized into hierarchical-based routing, flat-based routing, and location-based routing. In hierarchical-based routing, nodes assume diverse roles and functions in the network. In flat-based routing, all nodes perform identical roles. In location-based routing, the location of the sensor nodes is used for routing data in the network. When specific system parameters can be governed and controlled to adapt according to changes in the network and energy levels, then the routing protocols referred as adaptive protocols. Additionally, the adaptive routing protocols can be categorized into query-based, multipath-based, negotiation-based, coherent-based, QoS-based, routing protocols, based on the protocol operation.

Alternatively, based on the method of finding a route from source to destination, the routing protocols can be categorized into three types, reactive, proactive, and hybrid protocols. In reactive protocols, the routes will be established only when there is a requirement. In proactive protocols, all routes will be established and initialized at the beginning of network setup. The Hybrid protocols combine the concept of reactive and proactive protocols. Each of the protocols is suitable for few particular types of network. For example, proactive protocols such as table-driven protocols are suitable for static node network, as the nodes don't change the location and reactive protocol is unwanted. The reactive routing protocols consumea large amount of power to setup and initial new routes. The hybrid protocol tries to optimize and balance the energy utilization and routing efficiency.

Cooperative routing protocols are another type of routing protocols, wherein the nodes forward the data to a common node, known as acentral node. The central node accumulates the data and performs additional processing if required. This considerably reduces the energy utilization. On the other hand, several routing schemes depend on the location and timing information. Such protocols are discussed in later part of this paper. For simplicity, routing protocols based on network structure and protocol operation are considered for 


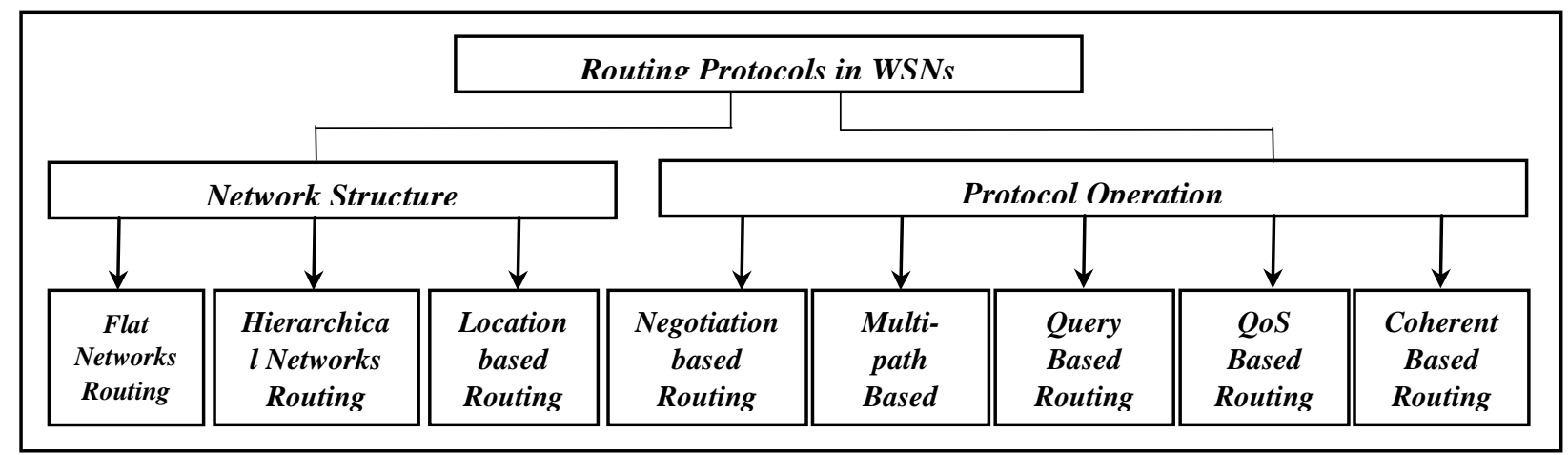

Figure 3: hierarchy of protocol classification

\section{Network Structure Based Protocols}

The operation of the routing protocol in WSNs is strongly determined by the underlying network structure. A detailed description of network structure based protocols is presented in this section.

\section{Flat Routing}

Multi-hop flat routing protocols are the first category of routing protocols. Each node in flat networks performs similar functions and roles. The sensor nodes work cooperatively tocarry out a given sensing task. As the number of nodes in such networks is quite high, it is not advisable to assign asingle identifier to all node. All nodes in this routing protocol will be given with same roles and functionalities. Since the nodes perform thesame function, they do not consider structural distribution. In such networks, bases station plays a major role to communicate with alarge array of nodes. Alternatively, node establishes direct and indirect communication with the base station. The node detects the closest neighboring nodes and forwards data on the basis of data-centric routing mode.

The flat routing is advantageous because it is modest and eliminates the complexity. It assists in network scaling, due to identical node roles and functions. By using the advantages of multi-hops routing, the network lifetime can be easily enhanced. The multi-hop routing balances the load and power utilization at the nodes during communication. On the downside, flat routing protocol does not ensure fairness among nodes. This might eventually result in theconcept of thehotspot when the nodes are homogeneously distributed in a WSN. In simple terms, let's say that there is only one sink node in the WSN, then all surrounding nodes of the sink node will expend large amounts of energy, which might degrade the network lifetime [47].
Some of the popular flat routing protocols are Sensor Protocols for Information via Negotiation (SPIN), Gossiping, Flooding, and Directed Diffusion (DD). SPIN and directed diffusion eradicate the existence of redundant data. It also employs data negotiation to minimize energy usage in WSN. Flooding forwards the data packets to all the nodes in the network. After receiving the data, the nodes forward again to other nodes in its neighborhood. Gossiping is slightly similar to flooding, but it chooses one node at random, to forward the data. This minimizes the energy consumption compared to flooding.

\section{Hierarchical-based routing}

In Hierarchical-based routing protocol, the nodes in a network are assigned with different functions, roles, and capabilities. The network is sub-divided to a number of clusters, in a process known as clustering. A cluster is composed of several grouped nodes, with a single nominated cluster head. All nodes in the cluster will forward the data to the cluster head. The cluster head aggregates all the data and forwards it to respective sink. Therefore, cluster head must perform collection and aggregation of data, transmission of aggregated data to thebase station, and managing the clusters. Preservation of Clusters is based on proficiency in identifying and recovering from cluster head failures. The selection and appointment of cluster heads will be done periodically to reduce the possibility of anodefailure and to maintain even utilization of energy. This additionally helps in extending the lifespan of the network. However, the cluster heads still end up using high amounts of energy. Hence, it is advisable to appoint new cluster heads periodically before a cluster head exhausts completely. This further eliminates the energy hotspot. Such energy efficiency measures in clustering technique help in maintaining good network scalability, enhanced lifespan and good performance [48]. 
Data aggregation is one of the foremost advantages of the hierarchical-based routing. The data aggregation takes place at thecluster head, which accumulates the data from all the member nodes, and remove any redundant data. Nevertheless, the hierarchical-based routing is not free from disadvantages. The protocol suffers from causing an energy hotspot at the cluster head selection. The cluster head needs ahigh amount of power. The complexity of deploying the network is high due to the overhead of maintaining a balance in energy utilization among the constituent nodes. In some cases, the messages overhead in clusters are also high.

There are numerous hierarchical-based routing in existence today, among which some of the commonly used protocols are, Minimum energy consumption network (MECN), Threshold sensitive Energy Efficient sensor Network protocol (TEEN), Adaptive Threshold sensitive Energy Efficient sensor Network protocol (APTEEN), Low-energy adaptive clustering hierarchy (LEACH), Power efficient Gathering in Sensor Information Systems (PEGASIS). MECN establishes a sub-network within the WSN, which contains alower number of nodes. It estimates the minimum global energy needed for data transfer. TEEN is designed to be reactive to random and radical variations in the sensed data, for instance, humidity, pressure, temperature, rainfall, etc. APTEEN is also reactive to such changes, but it focuses on collecting the data periodically. LEACH creates the clusters on the basis of received signal strength. It uses the native cluster heads as agateway to forward the data to the nearest base station. PEGASIS creates a chain of sensor nodes so that each node can send and receive from a neighboring node. It selects only one node from the chain to forward that aggregated data to the base station.

\section{Location-based routing}

In Location-based routing, the current location of the nodes is used for routing packets in the network. Every node in the network will be determined by its location. This information is used to detect, choose and retain optimal paths to transmit the data. The distance between nodes and potential energy requirement will be periodically calculated for this purpose. This further requires checking the location of thenode, regularly. The energy consumption can be minimized by using power management techniques, which can also enhance the network lifespan. Under power saving schemes, the unused or idle nodes can be put to sleep mode.

Location information and partitioning schemes are the main driving force behind such routing protocols. Using location information for routing purposes is advantageous because it helps in optimizing the management of network and minimizes the overhead of controlling the network [49]. Nevertheless, it is slightly difficult to design criteria and mechanism of sleep mode for the nodes. Greedy Perimeter Stateless Routing (GPSR) and Geographical and Energy Aware Routing (GEAR) are best examples of Location-based routing. In GPSR, an optimal route is discovered by using theperimeter of the planar graph. The location service in GPSR should map the locations and node identifiers. The main objective of GEAR is to optimize power consumption. It does so by setting an efficient forwarding route by using energy efficient and neighbors' location information.

\section{Protocol operation based routing}

The protocol operation determines the classification of these routing protocols. Various routing functionalities can be implemented based on the diversity of the technique used in the protocol. Ref. [49] discusses a detailed summary of the taxonomy of various operation based routing protocols, which are presented below.

Negotiation-based routing: This negotiation based routing protocol works on the principle of exchanging numerous negotiation messages between interconnected nodes. It helps in minimizing data redundancy and avoid creation of duplicate data [50]. Sensor Protocols for Information via Negotiation (SPIN) is the popular example of negotiation based routing protocol. The protocol uses negotiations to resolve the flooding issues. It utilizes meta-data to provide adetailed description of sensor data. Directed Diffusion (DD) employs flooding based query technique for uninterrupted and combined queries. Sequential assignment routing (SAR) is another such protocol, which generates multiple trees, each rooted at a 1 hop neighbor of the sink. This process creates more than one paths source to destination.

Multipath-based routing: This routing protocol focuses on determining other alternative paths between sources and destination, which are more efficient, in order to enhance the overall efficiency and minimize energy consumption in WSN. This protocol stipulates a minimum reserve energy level, which comes as an advantage to preserve the energy consumption and enhance the lifespan of the network [51]. It additionally reduces the risk of broken routes and enhances fault tolerance. The transmission delay can be reduced, which increases the network reliability. This subsequently results in greater network performance and ensures proper 


\section{Available online at www.ijrat.org}

communication. On the downside, this approach induces greater complexity on the network. It also increases energy consumption in the network, as it transmits periodic messages to check the path. Furthermore, establishing and maintaining all trees are not cheap. Some of the popular protocols of this category are, Multipath and Multi-SPEED (MMSPEED) and Sensor Protocols for Information via Negotiation (SPIN). The SPIN resolves the flooding issues through negotiations. The MMSPEED offers QoS differentiation in terms of reliability and time. It minimizes the protocol overhead.

Query-based routing: In this routing protocol, the transmission path is established based on a series of query packet exchanges between the sources and thedestination node. First, the destination node forwards a query of interest to a node in the network. When this query packet reaches the node of interest, it replies to the node which has initiated the query. The query is generally composed using high-level languages. By constantly updating the information related to each path in the network, an efficient route can be setup [52]. One of the foremost advantages of this protocol is it eradicated the data redundancy. It further minimizes the number of data transmissions in the network. On the downside, these protocols are not suitable for networks which require continuous data transmissions, such as surveillance and monitoring applications. Sensor Protocols for Information via

Coherent-based routing: This routing protocol is associated with two data processing approaches in WSN, namely, coherent and non- coherent schemes. Depending on the complexity of the processing, an energy-efficient route will be chosen for data transmission. In the coherent routing scheme, the data will be forwarded after some basic processing, in order to minimize the energy consumption. In non-coherent routing scheme, the data will be forwarded after complete local processing at each node [50]. The coherent scheme
Negotiation (SPIN) is the best example of Querybased routing. In this technique, the issues of flooding are resolved by constant negotiations, and it uses meta-data to provide a comprehensive description of sensor data. On the other hand, the Directed Diffusion (DD) technique employs flooding based query mechanism for continuous and collective queries.

QoS-based routing: This QoS based routing protocol works on creating a balance among all the network constraints to fulfill the QoS metrics. The most commonly considered network constraints are, data priority, data security, data quality, energy consumption, processing delay, bandwidth and so on [53]. Nevertheless, there is anapparent delay in fulfilling the QoS metrics, which degrades the overall performance and efficiency of this protocol. This eventually leads to higher energy utilization in the network. Additionally, it is expensive to create and retain all the trees in the network. Sequential assignment routing (SAR) is the best example of QoS-based routing scheme. It generates multiple trees, in which each branch creates a root to it 1hop neighbor of the sink. This task is performed to create more than one route from source to destination. This reduces the average weighted QoS metric over the network lifespan. Multipath and Multi-SPEED (MMSPEED) is another routing protocol, which provides QoS differentiation on the basis of reliability and time. This aide in reducing protocol overhead.

is more beneficial, as the preprocessing and data aggregation reduces the overall consumption of energy. In thenon-coherent scheme, target detection is required because data collection and preprocessing must be performed. For target detection, assistance is taken from the neighboring nodes, which requires thelatest information on the network topology. Then, a selection of central node is required to carry out data processing. On the downside, the central node should possess sufficient energy and other resources to carry out crucial task of computation.

\begin{tabular}{|l|l|l|c|l|c|c|c|c|}
\hline \multicolumn{1}{|c|}{$\begin{array}{c}\text { Routing } \\
\text { Technique }\end{array}$} & $\begin{array}{c}\text { Classificatio } \\
\mathrm{n}\end{array}$ & $\begin{array}{c}\text { Power } \\
\text { consumptio } \\
\mathrm{n}\end{array}$ & $\begin{array}{c}\text { Data } \\
\text { collectio } \\
\mathrm{n}\end{array}$ & $\begin{array}{c}\text { Scalabilit } \\
\text { y }\end{array}$ & $\begin{array}{c}\text { Quer } \\
\text { ybase } \\
\mathrm{d}\end{array}$ & $\begin{array}{c}\text { Comm. } \\
\text { Overhea } \\
\mathrm{d}\end{array}$ & $\begin{array}{c}\text { Data delivery } \\
\text { model }\end{array}$ & QoS \\
\hline SPIN & $\begin{array}{l}\text { Flat / Data } \\
\text { centric }\end{array}$ & Limited & $\checkmark$ & Limited & $\checkmark$ & Low & Event driven & $\mathbf{x}$ \\
\hline $\begin{array}{l}\text { Direct } \\
\text { Diffusion }\end{array}$ & $\begin{array}{l}\text { Flat / Data } \\
\text { centric }\end{array}$ & Limited & $\checkmark$ & Limited & $\checkmark$ & Low & $\begin{array}{l}\text { Demanddrive } \\
\mathrm{n}\end{array}$ & $\mathbf{x}$ \\
\hline $\begin{array}{l}\text { Rumor } \\
\text { Routing }\end{array}$ & Flat & Low & $\checkmark$ & Good & $\checkmark$ & Low & $\begin{array}{l}\text { Demanddrive } \\
\mathrm{n}\end{array}$ & $\mathbf{x}$ \\
\hline
\end{tabular}




\section{Available online at www.ijrat.org}

\begin{tabular}{|l|l|l|c|l|l|l|l|c|}
\hline $\begin{array}{l}\text { Gradientba } \\
\text { sed routing }\end{array}$ & Flat & Low & $\checkmark$ & Limited & $\checkmark$ & Low & Hybrid & $x$ \\
\hline CADR & Flat & Limited & $\checkmark$ & Limited & $\checkmark$ & Low & Continuously & $x$ \\
\hline COUGAR & Flat & Limited & $\checkmark$ & Limited & $\checkmark$ & High & Querydriven & $\mathbf{x}$ \\
\hline ACQUIRE & $\begin{array}{l}\text { Flat / Data } \\
\text { centric }\end{array}$ & Low & $\checkmark$ & Limited & $\checkmark$ & Low & $\begin{array}{l}\text { Complex } \\
\text { query }\end{array}$ & $\mathbf{x}$ \\
\hline LEACH & Hierarchical & High & $\checkmark$ & Good & $\mathbf{x}$ & High & Cluster head & $\mathbf{x}$ \\
\hline $\begin{array}{l}\text { TEEN \& } \\
\text { APTEEN }\end{array}$ & Hierarchical & High & $\checkmark$ & Good & $\mathbf{x}$ & High & $\begin{array}{l}\text { Active } \\
\text { threshold }\end{array}$ & $\mathbf{x}$ \\
\hline PEGASIS & Hierarchical & Max & $\mathbf{x}$ & Good & $\mathbf{x}$ & Low & Chains based & $\mathbf{x}$ \\
\hline VGA & Hierarchical & Low & $\checkmark$ & Good & $\mathbf{x}$ & High & Good & $\mathbf{x}$ \\
\hline SOP & Hierarchical & Low & $\mathbf{x}$ & Good & $\mathbf{x}$ & High & Continuously & $\mathbf{x}$ \\
\hline GAF & $\begin{array}{l}\text { Hierarchical } \\
\text { Location }\end{array}$ & Limited & $\mathbf{x}$ & Good & $\mathbf{x}$ & $\begin{array}{l}\text { Moderat } \\
\text { e }\end{array}$ & Virtual-grid & $\mathbf{x}$ \\
\hline SPAN & $\begin{array}{l}\text { Hierarchical } \\
\text { / Location }\end{array}$ & Limited & $\checkmark$ & Limited & $\mathbf{x}$ & High & Continuously & $\mathbf{x}$ \\
\hline GEAR & Location & Limited & $\mathbf{x}$ & Limited & $\mathbf{x}$ & $\begin{array}{l}\text { Moderat } \\
\text { e }\end{array}$ & $\begin{array}{l}\text { Demand } \\
\text { driven }\end{array}$ & $\mathbf{x}$ \\
\hline SAR & Data-centric & High & $\checkmark$ & Limited & $\checkmark$ & High & Continuously & $\checkmark$ \\
\hline SPEED & $\begin{array}{l}\text { Location/Da } \\
\text { tacentric }\end{array}$ & Low & $\mathbf{x}$ & Limited & $\checkmark$ & Less & Geographic & $\checkmark$ \\
\hline
\end{tabular}

Table 3: presents a comparative analysis of routing protocols, by considering few important factors for analytical comparison.

The compared routing protocols are, TEEN \& APTEEN ([Adaptive] Threshold sensitive Energy Efficient sensor Network), CADR (Constrained Anisotropic Diffusion Routing), GBR(Gradient Based Routing), DD(Directed Diffusion) ,RR(Rumor Routing), PEGASIS (The Power-Efficient Gathering in Sensor Information Systems), SOP(Self Organizing Protocol), GAF (Geographic Adaptive Fidelity), ACQUIRE (Active Query forwarding In sensor networks), SAR(Sequential Assignment Routing), LEACH(Low Energy Adaptive Clustering), VGA(Virtual Grid Architecture Routing), GEAR (Geographical and Energy Aware Routing), etc..

\subsection{Load balancing and clustering for lifetime maximization}

Sensors are small electronic transducers which possess the capacity to capture physical information like, light, heat, moisture, themotion of an object in an environment. When a particular region is intended to be monitored, then the sensors can be deployed randomly in an ad-hoc pattern.
Since its deployment, the sensors will be left unattended. They govern themselves and communicate with each other, which has a profound effect on the overall efficiency of the applications in consumer and military domains, such as, disaster management, battlefield surveillance, stock management, etc. In such application, the sensor is disposable, and they can be used as long as they have the power to work. Thus, the energy availability is inadequate for such sensor systems. Therefore, energy has to be properly managed to enhance the lifespan of the sensors, at least until the given objective is complete. In general, the sensor networks pursue after the model of a base station or command node. In this architecture, the sensors forward streams of data to the command node during a particular event, or just periodically. The command node will be present in the neighborhood of a node, or it can move along a given area to collect data from the sensor in its path. Either static or mobile, the command node is not easily accessible to the sensors. If the command node is located very far 
from a given sensor, then the sensor has to spend more energy to transmit the data to the node, which results in early depletion of energy [53].

A typical wireless sensor network is composed of a lot of sensor nodes which has limited resources and few other control nodes, which are slightly enhanced than normal nodes. Every sensor node contains a battery which acts as a source of energy, a low-end processor, and a small amount of storage and memory. They additionally contain a low power communication module with antenna for sending and receiving signals through awireless medium. The nodes are deployed randomly in the network, and lower battery back-up will eventually lead to node failure because the batteries cannot be replaced. Hence, energy must be spent carefully. A significant amount of energy is spent for data related processing, such as sensing, receiving, and forwarding and processing. Normally, a typical sensor node does not possess theadequate power to forward the data directly to the base station. Therefore, apart from sensing and sending the data, a node must also behave as a router to forward data from source to destination. If the sensor network is very large, the sensor nodes can be grouped to form smaller clusters. Each cluster contains acertain amount of member nodes and a cluster head to manage these nodes. Clustering is known to enhance the lifespan of thenetwork. It operates by a simple concept of aggregating the data and removing redundancy. This aggregated data can be forwarded as awhole in a single packet, directly to the base station.

Clustering approach also aides in creating agood balance of load distribution in the wireless sensor networks [54]. In a cluster-based load balancing mechanism [53], the cluster head is appointed based on the maximum transmission power of the nodes. In the proposed technique, the cluster membership is determined by the communication cost, and it does not consider the backup recovery. A typical load balanced clustering technique [55] utilizes combined weight value, which contains distance between the head and the member, along with the residual power to enhance the choice of thecluster member. In a multi-hop load balancing clustering technique, the layered approach is used for intra-cluster and intercluster communication. This technique assumes that the network is homogeneous. The network lifetime can be enhanced by even distribution of cluster heads, which can be accomplished by reconfiguring the cluster head in WSNs for better load balancing [56].

A novel load balancing scheduling mechanism for WSN was developed in ref. [57]. It makes use of optimal scheduling procedure for forwarding the packets which govern the time slot for transmitting the data packets to other recipient nodes. This technique offers theeven probability of packet loss for all constituent nodes. The balanced cost objective function can be used for optimum scheduling. In heterogeneous wireless sensor networks, secure load balancing is achieved through hierarchical data aggregation. In this technique, a pseudo sink is introduced for enhancing the accuracy of data and making efficient bandwidth usage in the network, which can translate to higher network lifetime. Pruning mechanism was used to develop a novel load balanced algorithm [58], which addresses the energy hotspot issues. In this algorithm, the load is balanced in the cluster using pruning mechanism. In the algorithm, the evaluation function is associated with pruning mechanism, which exploits theresidual energy of the nodes along with its location, and a number of cluster nodes.

Load balancing in energy-aware connected coverage algorithm [59] is accomplished by using sensing coverage and network connectivity by partitioning the given volume of nodes into smaller groups. For ensuring proper network connectivity, certain nodes in a subset are turned on. Nevertheless, the algorithm has the overhead to determine the presence of critical nodes. A Threshold-BasedPower-aware Load Balancing algorithm [60] offers anintra-network mechanism for scalable distributed control of energy utilization. However, game theory and market-based algorithms can also be used. It is assumed that the connection in the network is complete. A Load Balanced Clustering Algorithm [61] helps in balancing the load among cluster heads by considering the traffic load as an important factor. This algorithm uses centralized approach and it is an NP-hard. It assumes that all sensor nodes equally contribute to the traffic load. It believes that each node is aware of the network.

\section{Clustering objectives}

Different clustering techniques have different objectives. Most commonly, the clustering objective is set in order to facilitate meeting the requirements of the application. For instance, if an application is sensitive to data latency, then the length of data routes and inter/intraconnectivity must be taken in to account for clustering and selection of $\mathrm{CH}$ (cluster head). Some of the general objectives of network clustering are given below:

Load balancing: In general, the objective of clustered node setup demands uniform distribution of sensors nodes among the clusters. This ensures that there is an equal load on data processing or intra-cluster management duties among constituent $\mathrm{CH}$ [62]. With respect to roles and responsibilities 


\section{Available online at www.ijrat.org}

of $\mathrm{CHs}$, it is required to distribute the load equally among them to ensure that they fulfill QoS and other network performance goals [63]. Nevertheless, load balancing has turned out to be a critical problem in WSNs [64]. The selection of $\mathrm{CH}$ is based on certain criteria so that the $\mathrm{CH}$ doesn't fail during the network operation. Therefore, it is essential to construct clusters which have identical sizes. This avoids premature exhaustion of power and resources. But, equally distributing the sensors in a cluster causes additional delay [65]. When data aggregation is initiated by a $\mathrm{CH}$, it is advisable to have anidentical amount of nodes in the clusters, which can help in preparing collective data report simultaneously, for further processing of data at the next tier in the network.

Fault-tolerance: In general, the WSNs will be deployed in harsh and unknown environments and hence, the nodes might encounter theadditional risk of external attacks, physical damage, and general malfunction. Failure tolerance in $\mathrm{CHs}$ is highly significant in many applications, which has critical conditions over data integrity and communication. However, in worst cases of a $\mathrm{CH}$ failure, the recovery can be performed by re-clustering the network. Nevertheless, re-clustering not only imposes a burden on the resource of the nodes, it also causes trouble and disruption to the ongoing communication. In such cases, contemporary faulttolerance schemes are more suitable. This is done by appointing an auxiliary sensor node which can act as a backup $\mathrm{CH}$. The backup $\mathrm{CH}$ will be promoted to $\mathrm{CH}$ when the original $\mathrm{CH}$ fails. This ensures that any ongoing operations are unaffected. If any $\mathrm{CHs}$ have long radio range, then the neighboring $\mathrm{CHs}$ can look after the sensors in thecluster whose $\mathrm{CH}$ has failed [66]. Additionally, the role of $\mathrm{CH}$ can be rotated among the constituent nodes to ensure each node is utilized efficiently and guarantees alonger uptime of all the nodes.

Increased connectivity and reduced delay: Connectivity is a crucial requirement in numerous WSN applications, especially when $\mathrm{CHs}$ possess and theability of long-range communication, such as inter- $\mathrm{CH}$, satellite, etc. This holds correct while choosing a $\mathrm{CH}$ from available sensors population. The criteria of connectivity can be fulfilled by maintaining a free path form $\mathrm{CH}$ to the base-station [67]. It can also be accomplished by enforcing abound on the length of the path. When a node is

\begin{tabular}{|l|c|c|c|c|c|c|l|}
\hline $\begin{array}{c}\text { Clustering } \\
\text { Technique }\end{array}$ & $\begin{array}{c}\text { Node } \\
\text { Mobility }\end{array}$ & $\begin{array}{c}\text { Cluster } \\
\text { overlapping }\end{array}$ & $\begin{array}{c}\text { Location } \\
\text { awareness } \\
\text { requirement }\end{array}$ & $\begin{array}{c}\text { Energy } \\
\text { efficient }\end{array}$ & $\begin{array}{c}\text { Failure } \\
\text { recovery }\end{array}$ & $\begin{array}{c}\text { Balanced } \\
\text { clustering }\end{array}$ & $\begin{array}{c}\text { Cluster } \\
\text { stability }\end{array}$ \\
\hline LCA & $\checkmark$ & $\times$ & $\checkmark$ & $x$ & $\checkmark$ & Medium & Moderate \\
\hline $\begin{array}{l}\text { Adaptive } \\
\text { Clustering }\end{array}$ & $\checkmark$ & $\times$ & $\checkmark$ & N/A & $\checkmark$ & Medium & Low \\
\hline
\end{tabular}

assigned with the task of $\mathrm{CH}$, the connectivity objective become one of theconnected dominating set problems in the network. Alternately, when delays are considered, the intra-cluster connectivity is perceived as a limitation. Delay can be factored in by configuring an upper limit on the number of hops " $\mathrm{K}$ " permitted on a given data path. K-hop clustering is K-dominating set problem.

Minimal cluster count: When the $\mathrm{CHs}$ are rich with underlying resources, the minimal cluster count is determined [68]. The network administrator is generally inclined towards creating a minimal number of cluster of these nodes, as they can be highly expensive and susceptible to attacks. For instance, if laptops, mobile devices, or robots, etc., are made $\mathrm{CHs}$, then there will be acertain restriction on a total number of nodes. The complexity and challenges in deploying the nodes can cause such restrictions. For instance, a WSN implemented to work in battlefield or forest. Additionally, these nodes have alarger size compared to other sensors, which is easier to identify them. In some WSN application like military reconnaissance, border surveillance, infrastructure security, etc., the node visibility is not anticipated.

Maximal network longevity: Network lifespan is a major concern in many application because of the limited resources of nodes. The nodes have limited energy supply which only lasts for short span of time. Moreover, the energy usage increases when the nodes are deployed in a harsh environment. When the resources of $\mathrm{CH}$ are found be higher than that of sensors, then the energy usage for intracluster communication must be reduced [69]. The placing of sensors must be closer to the $\mathrm{CH}$ [70]. In some cases, the $\mathrm{CH}$ will act as sensors too. In this situation, the uptime of $\mathrm{CH}$ can be extended by minimizing the load applied to the $\mathrm{CH}$. In general, thelifespan of a network can be extended by combining clustering and route establishment. Additionally, network life can be prolonged by using adaptive clustering too.

Depending on the clustering technique, numerous approaches have been presented to enhance the overall efficiency and performance of communication in WSN. Table 4 presents the comparative analysis of popular clustering schemes. 


\section{Available online at www.ijrat.org}

\begin{tabular}{|l|c|c|c|c|c|l|l|}
\hline CLUBS & $\checkmark$ & High & $x$ & N/A & $\checkmark$ & Medium & Moderate \\
\hline $\begin{array}{l}\text { Hierarchical } \\
\text { control } \\
\text { clustering }\end{array}$ & $\checkmark$ & Low & $x$ & N/A & $\checkmark$ & Good & Moderate \\
\hline GS3 & $\checkmark$ & Low & $\checkmark$ & N/A & $\checkmark$ & Good & Moderate \\
\hline RCC & $\checkmark$ & $x$ & $\checkmark$ & N/A & $\checkmark$ & Good & Moderate \\
\hline EEHC & $\checkmark$ & $x$ & $\checkmark$ & $\checkmark$ & N/A & Medium & N/A \\
\hline LEACH & Fixed BS & $x$ & $x$ & $x$ & $\checkmark$ & Medium & Moderate \\
\hline FLOC & $\checkmark$ & $x$ & $x$ & N/A & $\checkmark$ & Good & High \\
\hline ACE & $\checkmark$ & Low & $x$ & $x$ & $\checkmark$ & Good & High \\
\hline HEED & Stationary & $x$ & $x$ & $\checkmark$ & N/A & Good & High \\
\hline Extended HEED & Stationary & $x$ & $x$ & $\checkmark$ & N/A & Very good & High \\
\hline DWEHC & Stationary & $x$ & $\checkmark$ & $\checkmark$ & N/A & Very good & High \\
\hline MOCA & Stationary & $\checkmark$ & $x$ & $\checkmark$ & N/A & Good & High \\
\hline $\begin{array}{l}\text { Attribute based } \\
\text { clustering }\end{array}$ & $x$ & $x$ & $\checkmark$ & $\checkmark$ & $\checkmark$ & Very good & High \\
\hline
\end{tabular}

Table 4: load balancing approaches

\section{CONCLUSION}

The demandfor wireless communication is increasing rapidly. In this field of wireless communication, wireless sensor networks play animportant rolein providing efficient wireless communication. These communication models are adopted for areal-time communication system where better quality-of-service is demanded for each communication period. However, various researchhas been carried out in this field of WSN communication which helps to provide reliable and better communication. Recent advancements in technology have increased the utilization of wireless communication which causes congestion in network resulting in degraded WSN communication. Recent studies show that the performance of WSN can be improved further by improving its process of data collection, data transmission, and energy efficient communication. In this article, we have focused on WSN communication efficiency and presented a comparative study on WSN localization, routing,and load balancing. This complete study shows that performance can be improved further.
Based on this comparative survey study, in future we will try to resolve various issues of wireless sensor network which are as follows:

(a) Data collection is an important task in this field which can be derived by developing WSN localization scheme. In future work, we will focus on development and implementation of faulttolerant WSN localization scheme for reliable communication without packet drop.

(b) The study shows that WSN routing plays an important role to improve the performance of WSN, recently, energy-aware routing techniques have been discussed and employed in WSN research field to enhance the performance, however, these techniques suffer from the complexity, pack drop issues. To overcome this, we will focus on the bio-inspired routing techniques which can be useful for better QoS.

(c) In the next phase, we will focus on network lifetime enhancement by developing theloadbalancing algorithm. To overcome the issues of network lifetime, we will present a fault-tolerant clustering algorithm for lifetime enhancement where node clustering, route recovery, and data traffic will be considered to make it more adaptive for any environment. 


\section{REFERENCES}

[1] I.F. Akyildiz, Weilian Su, Y. Sankarasubramaniam, and E. Cayirci, "A survey on sensor networks," IEEE Communications Magazine, vol. 40, no. 8, pp. 102 -114, August 2002.

[2] Ghoreyshi, S.M., Shahrabi, A. and Boutaleb, T., 2017. Void-Handling Techniques for Routing Protocols in Underwater Sensor Networks: Survey and Challenges. IEEE Communications Surveys \& Tutorials, 19(2), pp.800-827.

[3] Liu, J., Shen, H., Yu, L., Narman, H.S., Zhai, J., Hallstrom, J.O. and He, Y., 2017. Characterizing data deliverability of greedy routing in wireless sensor networks. IEEE Transactions on Mobile Computing.

[4] Boyle, D., Kolcun, R. and Yeatman, E., 2017. Energy-Efficient Communication in Wireless Networks. In ICT-Energy Concepts for Energy Efficiency and Sustainability. InTech.

[5] Zhu, Y. and Ni, L.M., 2016. A probabilistic approach to statistical QoS provision of event detection in sensor networks. Wireless Networks, 22(2), pp.439-451.

[6] Kashi, S.S., 2017. A load balanced location service for location information management of multi-sink Wireless Sensor Networks. Computing, pp.1-25.

[7] Bulusu, N., Heidemann, J. and Estrin, D., 2000. GPS-less low-cost outdoor localization for very small devices. IEEE personal communications, 7(5), pp.28-34.

[8] Simonetto, A. and Leus, G., 2014. Distributed Maximum Likelihood Sensor Network Localization. IEEE Trans. Signal Processing, 62(6), pp.1424-1437.

[9] Xiong, J. and Jamieson, K., 2013. Arraytrack: A fine-grained indoor location system. Usenix.

[10] Alrajeh, N.A., Bashir, M. and Shams, B., 2013. Localization techniques in wireless sensor networks. International Journal of Distributed Sensor Networks, 9(6), p.304628.

[11] He, T., Huang, C., Blum, B.M., Stankovic, J.A. and Abdelzaher, T., 2003, September. Range-free localization schemes for large scale sensor networks. In Proceedings of the 9th annual international conference on Mobile computing and networking (pp. 81-95). ACM.

[12] Mármol, F.G. and Pérez, G.M., 2011. Providing trust in wireless sensor networks using a bioinspired technique. Telecommunication systems, 46(2), pp.163-180.

[13] Alzaid H., Alfaraj M., Ries S., Jøsang A., Albabtain M., Abuhaimed A. (2013) ReputationBased Trust Systems for Wireless Sensor Networks: A Comprehensive Review. In: Fernández-Gago C., Martinelli F., Pearson S.,
Agudo I. (eds) Trust Management VII. IFIPTM 2013.

[14]Baroutis, N. and Younis, M., 2017. Loadconscious maximization of base-station location privacy in wireless sensor networks. Computer Networks, 124, pp.126-139.

[15] Alrajeh, N.A., Bashir, M. and Shams, B., 2013. Localization techniques in wireless sensor networks. International Journal of Distributed Sensor Networks, 9(6), p.304628.

[16] Sichitiu, M.L. and Ramadurai, V., 2004, October. Localization of wireless sensor networks with a mobile beacon. In Mobile Ad-hoc and Sensor Systems, 2004 IEEE International Conference on (pp. 174-183). IEEE.

[17] Chen, H., Lou, W., Wang, Z., Wu, J., Wang, Z. and Xia, A., 2015. Securing DV-Hop localization against wormhole attacks in wireless sensor networks. Pervasive and Mobile Computing, 16, pp.22-35.

[18] Xu, S. and Dogancay, K., 2017. Optimal Sensor Placement for 3D Angle-of-Arrival Target Localization. IEEE Transactions on Aerospace and Electronic Systems.

[19] Wielandt, S., Shah, M.V., Athaullah, N.A., Sayyad, U.M., Yadav, R.B., Dharamdasani, R.M., De Strycker, L. and Kuhn, M., 2017, March. 2.4 $\mathrm{GHz}$ single anchor node indoor localization system with angle of arrival fingerprinting. In Wireless Days, 2017 (pp. 152-154). IEEE.

[20] Ren, Y., Salim, F.D., Tomko, M., Bai, Y.B., Chan, J., Qin, K.K. and Sanderson, M., 2017. DLog: A WiFi Log-based differential scheme for enhanced indoor localization with single RSSI source and infrequent sampling rate. Pervasive and Mobile Computing, 37, pp.94-114.

[21] Xiong, J., Qin, Q. and Zeng, K., 2014, December. A distance measurement wireless localization correction algorithm based on RSSI. In Computational Intelligence and Design (ISCID), 2014 Seventh International Symposium on (Vol. 2, pp. 276-278). IEEE.

[22] Abdullah, H., Khan, I. and Zainal, M.S., 2014, March. Improved range estimation method in WSN for human intruder localization. In Signal Processing \& its Applications (CSPA), 2014 IEEE 10th International Colloquium on (pp. 87-90). IEEE.

[23] Maddumabandara, A., Leung, H. and Liu, M., 2015. Experimental evaluation of indoor localization using wireless sensor networks. IEEE Sensors Journal, 15(9), pp.5228-5237.

[24] Mekonnen, Z.W. and Wittneben, A., 2014, September. Robust TOA based localization for wireless sensor networks with anchor position uncertainties. In Personal, Indoor, and Mobile Radio Communication (PIMRC), 2014 IEEE 25th 


\section{Available online at www.ijrat.org}

Annual International Symposium on (pp. 20292033). IEEE.

[25] Xiong, H., Chen, Z., An, W. and Yang, B., 2015. Robust TDOA localization algorithm for asynchronous wireless sensor networks. International Journal of Distributed Sensor Networks, 11(5), p.598747.

[26] Singh, P. and Agrawal, S., 2013, April. TDOA based node localization in WSN using neural networks. In Communication Systems and Network Technologies (CSNT), 2013 International Conference on (pp. 400-404). IEEE.

[27] Hui, X., Zhiyuan, C., Beiya, Y. and Rongpei, N., 2015. TDoA localization algorithm with compensation of clock offset for wireless sensor networks. China Communications, 12(10), pp.193-201.

[28]Zhao, L.Z., Wen, X.B. and Li, D., 2014. Amorphous localization algorithm based on BP artificial neural network.

[29]Zhao, C., Xu, Y. and Huang, H., 2014. Weighted centroid localization based on compressive sensing. Wireless networks, 20(6), pp.1527-1540.

[30] Kumar, S. and Lobiyal, D.K., 2013. An advanced DV-Hop localization algorithm for wireless sensor networks. Wireless personal communications, pp.1-21.

[31] Singh, S.P. and Sharma, S.C., 2015. Range free localization techniques in wireless sensor networks: A review. Procedia Computer Science, 57, pp.7-16.

[32]Zhang, X., Xiong, W. and Xu, B., 2017. A Computationally Efficient Received Signal Strength Based Localization Algorithm in ClosedForm for Wireless Sensor Network. Neural Processing Letters, pp.1-15.

[33] L. Heng and G. Gao, "Accuracy of Range-Based Cooperative Positioning: A Lower Bound Analysis," in IEEE Transactions on Aerospace and Electronic Systems, vol. PP, no. 99, pp. 1-1

[34]Zhao, Y., Liu, K., Ma, Y., Gao, Z., Zang, Y. and Teng, J., 2017. Similarity Analysis-Based Indoor Localization Algorithm With Backscatter Information of Passive UHF RFID Tags. IEEE Sensors Journal, 17(1), pp.185-193.

[35] Li, P., Yu, X., Xu, H., Qian, J., Dong, L. and Nie, H., 2017. Research on secure localization model based on trust valuation in wireless sensor networks. Security and Communication Networks, 2017.

[36] Singh, M. and Khilar, P.M., 2017. Mobile beacon based range free localization method for wireless sensor networks. Wireless Networks, 23(4), pp.1285-1300.

[37] Porta, J.M., Rull, A. and Thomas, F., 2016. Sensor Localization from Distance and Orientation Constraints. Sensors, 16(7), p.1096.
[38] Pivato, P., Palopoli, L. and Petri, D., 2011. Accuracy of RSS-based centroid localization algorithms in an indoor environment. IEEE Transactions on Instrumentation and Measurement, 60(10), pp.3451-3460.

[39] Zhang, S., Liu, X., Wang, J., Cao, J. and Min, G., 2015. Accurate range-free localization for anisotropic wireless sensor networks. ACM Transactions on Sensor Networks (TOSN), 11(3), p.51.

[40] Kwon, Y., Mechitov, K. and Agha, G., 2014. Design and implementation of a mobile actor platform for wireless sensor networks. In Concurrent objects and beyond (pp. 276-316). Springer Berlin Heidelberg.

[41] Tran, D.A. and Nguyen, T., 2008. Localization in wireless sensor networks based on support vector machines. IEEE Transactions on Parallel and Distributed Systems, 19(7), pp.981-994.

[42]Z. Li, W. Trappe, Y. Zhang, et al. Robust statistical methods for securing wireless localization in sensor networks[C]//Proceedings of the Fourth International Symposium on Information Processing in Sensor Networks. New York: ACM, 2005: $91-98$.

[43] L. Lazos, R. Poovendran. SeRLoc: Robust localization for wireless sensor networks [J]. ACM Transactions on Sensor Networks, 2005, 1(1): $73-100$.

[44] N. Bulusu, J. Heidemann, D. Estrin, “GPS-less low cost outdoor localization for very small devices", Technical report 00-729, Computer science department, University of Southern California, Apr. 2000

[45] W. Heinzelman, A. Chandrakasan and $H$. Balakrishnan, "Energy-Efficient Communication Protocol for Wireless Microsensor Networks," Proceedings of the 33rd Hawaii International Conference on System Sciences (HICSS '00), January 2000

[46] Y. Yao and J. Gehrke, "The cougar approach to in-network query processing in sensor networks", in SIGMOD Record, September 2002.

[47] Datta, N. N., \& Gopinath, K. (2006)"A survey of routing algorithms for wireless sensor networks", Journal of the Indian Institute of Science, Vol. 86, pp. 569-598.

[48] Nayak, P. and Vathasavai, B., 2017. Energy Efficient Clustering Algorithm for Multi-Hop Wireless Sensor Network Using Type-2 Fuzzy Logic. IEEE Sensors Journal, 17(14), pp.44924499.

[49] Al-Karaki, Jamal N., and Ahmed E. Kamal (2004)"Routing techniques in wireless sensor networks: a survey", IEEE Wireless Communications, Vol.11, No. 6, pp. 6-28.

[50] Pantazis, N.A., Nikolidakis, S.A. and Vergados, D.D., 2013. Energy-efficient routing protocols in 


\section{Available online at $w w w . i j r a t . o r g$}

wireless sensor networks: A survey. IEEE Communications surveys \& tutorials, 15(2), pp.551-591.

[51] Leu, J.S., Chiang, T.H., Yu, M.C. and Su, K.W., 2015. Energy efficient clustering scheme for prolonging the lifetime of wireless sensor network with isolated nodes. IEEE communications letters, 19(2), pp.259-262.

[52] Braginsky, D., \& Estrin, D. (2002) "Rumour routing algorithm for sensor networks", in Proceedings of the First Workshop on Sensor Networks and Applications (WSNA), pp. 22-31.

[53] Turkanović, M., Brumen, B. and Hölbl, M., 2014. A novel user authentication and key agreement scheme for heterogeneous ad hoc wireless sensor networks, based on the Internet of Things notion. Ad Hoc Networks, 20, pp.96-112.

[54] Lee, J.H. and Moon, I., 2014. Modeling and optimization of energy efficient routing in wireless sensor networks. Applied Mathematical Modelling, 38(7), pp.2280-2289.

[55]H. Zhang, L. Li, X. Yan, X. Li, "A Load Balancing Clustering Algorithm of WSN for Data Gathering", 978-1-4577-0536-6/11, 2011, IEEE.

[56] N. Kim, J. Heo, H. S. Kim \& W. H. Kwon, "Reconfiguration of Cluster head for Load Balancing in Wireless Sensor Networks", Science Direct Computer Communications 31 (2008) 153159

[57] Souissi, M. and Meddeb, A., 2017, January. Modelling of clustering with relay nodes in wireless sensor networks. In Computing and Communication Workshop and Conference (CCWC), 2017 IEEE 7th Annual (pp. 1-6). IEEE.

[58] Y. Zhang, Z. Zheng, Y. Jin, X. Wang," Load Balanced Algorithm In Wireless Sensor Networks Based on Pruning Mechanism", IEEE transaction, 978-0-7695-3522-7/09.

[59] M. Mahdari, M. Ismail, K. Jumari, "Load Balancing in Energy Efficient Connected Coverage Wireless Sensor Networks", IEEE Transaction, Volume: 02 pp. 448-452, 2009

[60] C. M. Canci, V. Trifa \& A. Martinoli, "Threshold Based Algo. For Power Aware Load Balancing in Sensor Networks", IEEE Transaction, 0-78038916-6/05, 2005.

[71] ss Networks. IEEE/ACM Transactions on Networking.
[61] C.P.Low, C. Fang, J. Mee, Ng \& Y.H. Hang, "Load Balanced Clustering Algorithm for Wireless Sensor Networks", IEEE Communications Society, 1-4244-0353-7/07, 2007.

[62] G. Gupta, M. Younis, Load-balanced clustering in wireless sensor networks, in: Proceedings of the International Conference on Communication (ICC 2003), Anchorage, Alaska, May 2003.

[63] Abbasi, A.A. and Younis, M., 2007. A survey on clustering algorithms for wireless sensor networks. Computer communications, 30(14), pp.2826-2841.

[64] Younis, O. and Fahmy, S., 2004. HEED: a hybrid, energy-efficient, distributed clustering approach for ad hoc sensor networks. IEEE Transactions on mobile computing, 3(4), pp.366-379.

[65] Oren, G., Barenboim, L. and Levin, H., 2017, June. Adaptive Distributed Hierarchical Sensing algorithm for reduction of wireless sensor network cluster-heads energy consumption. In Wireless Communications and Mobile Computing Conference (IWCMC), 2017 13th International (pp. 980-986). IEEE.

[66] G. Gupta, M. Younis, Fault-tolerant clustering of wireless sensor networks, in: Proceedings of the IEEE Wireless Communication and Networks Conference (WCNC 2003), New Orleans, Louisiana, March 2003

[67]F. Garcia, J. Solano, I. Stojmenovic, Connectivity based k-hop clustering in wireless networks, Telecommunication Systems 22 (1) (2003) 205220.

[68] Vincze, Z., Vida, R. and Vidacs, A., 2007, July. Deploying multiple sinks in multi-hop wireless sensor networks. In Pervasive Services, IEEE International Conference on (pp. 55-63). IEEE.

[69] Liu, C.M., Lee, C.H. and Wang, L.C., 2007. Distributed clustering algorithms for datagathering in wireless mobile sensor networks. Journal of parallel and distributed computing, 67(11), pp.1187-1200.

[70] Gao, X., Zhu, X., Li, J., Wu, F., Chen, G., Du, D.Z. and Tang, S., 2017. A Novel Approximation for Multi-Hop Connected Clustering Problem in Wirele 